\title{
Electron Acceleration during Macroscale Magnetic Reconnection
}

\author{
H. Arnold@, J. F. Drake, and M. Swisdak@ \\ IREAP, University of Maryland, College Park, Maryland 20742-3511, USA \\ F. Guo \\ Los Alamos National Lab, Los Alamos, New Mexico 87545, USA \\ J. T. Dahlin (1) \\ NASA Goddard Space Flight Center, Greenbelt, Maryland 20771, USA \\ B. Chen $\odot$ and G. Fleishman $\odot$ \\ New Jersey Institute of technology, Newark, New Jersey 07102, USA \\ L. Glesener \\ University of Minnesota, Minneapolis, Minnesota 55455, USA \\ E. Kontar \\ University of Glasgow, Glasgow G12 8QQ, United Kingdom \\ T. Phan \\ University of California, Berkeley, California 94720, USA \\ C. Shen $\odot$ \\ Harvard University, Cambridge, Massachusetts 02138, USA
}

(Received 10 November 2020; revised 19 January 2021; accepted 1 March 2021; published 30 March 2021)

\begin{abstract}
The first self-consistent simulations of electron acceleration during magnetic reconnection in a macroscale system are presented. Consistent with solar flare observations, the spectra of energetic electrons take the form of power laws that extend more than two decades in energy. The drive mechanism for these nonthermal electrons is Fermi reflection in growing and merging magnetic flux ropes. A strong guide field suppresses the production of nonthermal electrons by weakening the Fermi drive mechanism. For a weak guide field the total energy content of nonthermal electrons dominates that of the hot thermal electrons even though their number density remains small. Our results are benchmarked with the hard x-ray, radio, and extreme ultraviolet observations of the X8.2-class solar flare on September 10, 2017.
\end{abstract}

DOI: 10.1103/PhysRevLett.126.135101

Introduction.-Flares are explosive events in the solar corona that convert magnetic energy into particle energy through magnetic reconnection [1-4]. While some released energy goes into bulk flows and thermal energy, a significant fraction appears in nonthermal electrons, which have a power-law tail in their distribution function [2,4,5]. This can lead to their pressure approaching the ambient magnetic [6,7]. Observations by the Reuven Ramaty High Energy Solar Spectroscopic Imager (RHESSI) and the Atmospheric Imaging Assembly on the Solar Dynamic Observatory, suggest that these electrons comprise a significant fraction of the total electron density in the above-the-loop-top sources in solar flares [8].

Magnetic reconnection creates bent field lines with a tension that drives an Alfvénic exhaust away from the $\mathrm{x}$-line $[9,10]$, energizing the surrounding plasma. When particles stream into the exhaust, they gain energy by reflecting from bent field lines [11]. This process, Fermi reflection, is likely responsible for producing the powerlaw tails in the electron distribution function [11-14]. The energy gain due to Fermi reflection is proportional to a particle's energy and therefore dominates the energy gain of the most energetic electrons. Since Fermi reflection does not depend on any kinetic length scale, the relevant length is the curvature of the reconnecting magnetic field. Electrons can continue to gain energy from Fermi reflection even as flux ropes merge and approach the domain size [15].

This Letter presents the first results of simulations of particle acceleration during magnetic reconnection in a 2D macrosystem that includes the self-consistent feedback of energetic particles on the dynamics while conserving the 
global energy. The simulation model, kglobal [16,17], retains Fermi reflection as the dominant drive mechanism for energetic electrons but excludes parallel electric fields in kinetic-scale boundary layers. The kinetic scales that constrain PIC modeling of macroscale systems are eliminated. While the ordering of kglobal eliminates kineticscale parallel electric fields, the model includes large-scale parallel electric fields, important for the initial energy gain of electrons during reconnection [18-20].

The kglobal simulations produce power-law spectra of energetic electrons that extend nearly three decades in energy and simultaneously produce super-hot thermal electrons, consistent with flare observations [6,7,21]. The total energy content of the nonthermal electrons can exceed that of the hot thermal electrons even though the number density is smaller. Simulations performed with differing values of the initial ambient out-of-plane guide field, $B_{g}$ reveal that its magnitude strongly impacts the energy content of the nonthermal electrons and their power-law index. Stronger $B_{g}$ suppresses production of nonthermal electrons by weakening the Fermi drive mechanism. In contrast, the size of the global system has relatively little influence.

Numerical simulations setup.-The 2D simulations are carried out with the kglobal model, which consists of a magnetohydrodynamic (MHD) backbone with fluid ions and electrons as well as particle electrons that are distributed as macroparticles on the MHD grid. The two electron species combine so that charge neutrality is preserved $[16,17]$. However, since the equations governing energy gain in the electron fluid are incomplete (e.g., Fermi reflection is not included), any change in the energy of the electron fluid will be neglected in the analysis of the electron energy gain. The upstream reconnection magnetic field $B_{0}$, and the ion density $n_{0}$, define the Alfvén speed, $C_{A 0}=B_{0} / \sqrt{4 \pi m_{i} n_{0}}$. Since no kinetic scales are resolved, lengths are normalized to an arbitrary macroscale $L_{0}$. Times are normalized to $\tau_{A}=L_{0} / C_{A 0}$ and temperatures and particle energies to $m_{i} C_{A 0}^{2}$. The perpendicular electric field follows an MHD scaling, $C_{A 0} B_{0} / c$. The parallel electric field scales like $m_{i} C_{A 0}^{2} / e L_{0}$ and is small compared with the perpendicular component. However, the energy associated with the parallel potential drop acting over the scale $L_{0}$ is of order $m_{i} C_{A 0}^{2}$, which is comparable to the available magnetic energy per particle.

The simulations are initialized with constant densities and pressures in a force-free current sheet with periodic boundary conditions. Thus, $\boldsymbol{B}=B_{0} \tanh (y / w) \hat{\boldsymbol{x}}+$ $\sqrt{B_{0}^{2} \operatorname{sech}^{2}(y / w)+B_{g}^{2}} \hat{z}$. The temperatures of all three species are uniform and isotropic with $T_{i}=T_{e \text {,part }}=$ $T_{e, \text { fluid }}=0.0625 m_{i} C_{A 0}^{2}$, leading to an initial plasma $\beta$ of 0.25 (based on $B_{0}$ ). While the initial $\beta$ is higher than typical coronal values, electron heating, and acceleration is insensitive to this choice as well as to the chosen fraction of particle electrons (25\% of the total electrons). The domain size for all simulations is $L_{x} \times L_{y}=2 \pi L_{0} \times \pi / 2 L_{0}$. The magnetic field evolution equation includes a hyperresistivity $\nu$ to facilitate reconnection, while minimizing dissipation at large scales [22]. The effective Lundquist number $S_{\nu}=C_{A} L_{0}^{3} / \nu$ associated with this hyperresistivity is varied to change the effective system size (ratio of the macro to the dissipation scale). We also include fourth and second order viscosity terms and some electron particle diffusion to prevent a numerical instability associated with trapping electrons in small perpendicular electric field fluctuations. Reconnection begins from particle noise and proceeds to produce multiple flux ropes whose number increases with $S_{\nu}$. However, our late-time results are relatively insensitive to $S_{\nu}$ and therefore the effective system size. Unless otherwise stated we focus on simulations with 100 particles per cell, time step $d t=0.0001 \tau_{0}$, $S_{\nu}=9.5 \times 10^{7}$, and $N_{x} \times N_{y}=2048 \times 512$ grid cells. By taking $L_{0}=10^{4} \mathrm{~km}$, our grid cell is $30 \mathrm{~km}$ across, much larger than any kinetic scales or PIC simulations. The mass ratio is $m_{i} / m_{e}=25$. The results are not sensitive to this value. The speed of light is $c / C_{A 0} \approx 60$. We use guide fields $B_{g} / B_{0}=0.1,0.25,0.4,0.5,0.6,0.8$, and 1.0.

Simulation results.-Since reconnection in our simulations is triggered by particle noise, the dynamics begins with the growth of many small islands, which subsequently undergo mergers and eventually approach the system scale. This behavior is seen in Fig. 1 from a simulation with $B_{g} / B_{0}=0.25$. The energy per particle of the particle electrons, $\langle W\rangle$ (energy density divided by number density), is shown in the $x-y$ plane at three times, $t / \tau_{A}=2.5,5$, and 8 in panels (a),(b), and (c). Magnetic field lines are

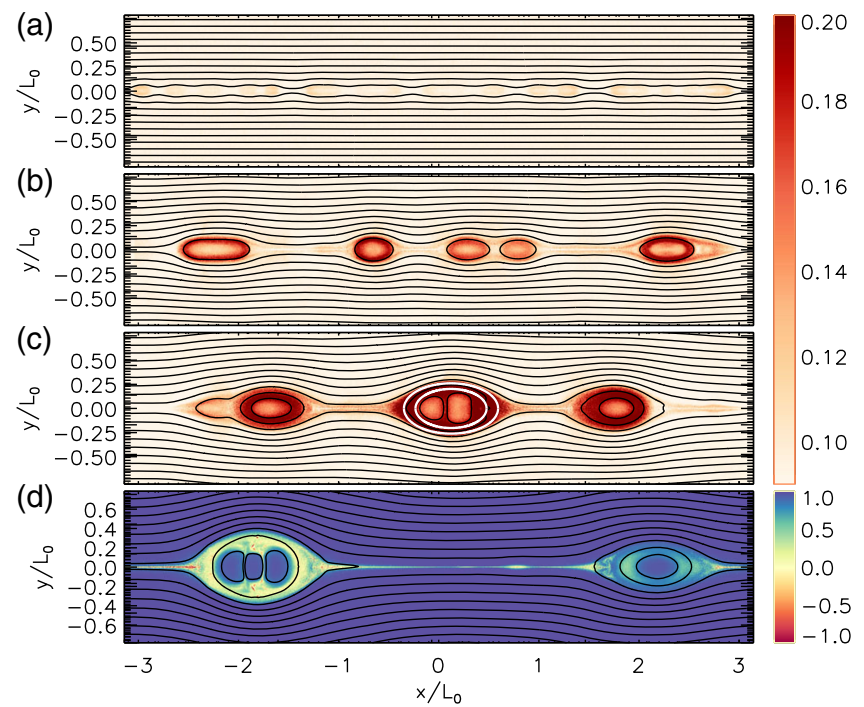

FIG. 1. (a),(b), and (c) $\langle W\rangle$ for a simulation with $B_{g} / B_{0}=0.25$ at $t / \tau_{A}=2.5,5$, and 8 with field lines overplotted. (d) The firehose parameter at late time from a simulation with $B_{g} / B_{0}=0.1$. 
superimposed. The particle electron energy is nearly constant along field lines because of the high electron parallel mobility.

Figure 1(d) shows the firehose parameter, $1-4 \pi\left(P_{\|}-\right.$ $\left.P_{\perp}\right) / B^{2}$ at late time from a simulation with $B_{g} / B_{0}=0.1$. Large regions within the magnetic islands are near marginal stability (with some unstable regions) so that the local magnetic tension, which drives particle energy gain, is largely suppressed there. Thus, electron feedback on the MHD fluid is essential in regimes where electron energy gain is significant. Models based on test particle dynamics neglect the feedback of particles and can therefore lead to runaway electron energy gain.

The spectrum of nonthermal electrons is calculated by summing the number of particle electrons within a specified energy range over the entire simulation domain. This improves the statistics by maximizing the count rate at the highest energies. Figure 2(a) shows the differential electron number density $F(W)=d N(W) / d W$ versus the normalized energy, $W / m_{i} C_{A 0}^{2}$, on a log-log scale at several times for the case $B_{g} / B_{0}=0.25$ shown in Fig. 1. $F(W)$ takes the form of a power law (a straight line in the log-log plot) as time progresses. The power-law index $\delta^{\prime}$ reaches a constant value at low energy early in time and later extends to higher energy.

The inset in Fig. 2 shows the late-time $F(W)$ for several values of $B_{q}$ corresponding to times when approximately the same amount of magnetic flux has reconnected. As $B_{g}$ decreases, $\delta^{\prime}$ decreases so the spectrum becomes harder and more high-energy electrons are produced. In Fig. 2(b) we plot the late time spectrum of $F(W)$ for several values of $S_{\nu}$. Larger values of $S_{\nu}$ correspond to larger systems. Thus, Fig. 2(b) demonstrates that the slope of the power law is relatively insensitive to the size of the system. However, the total energy contained in the nonthermal electrons increases

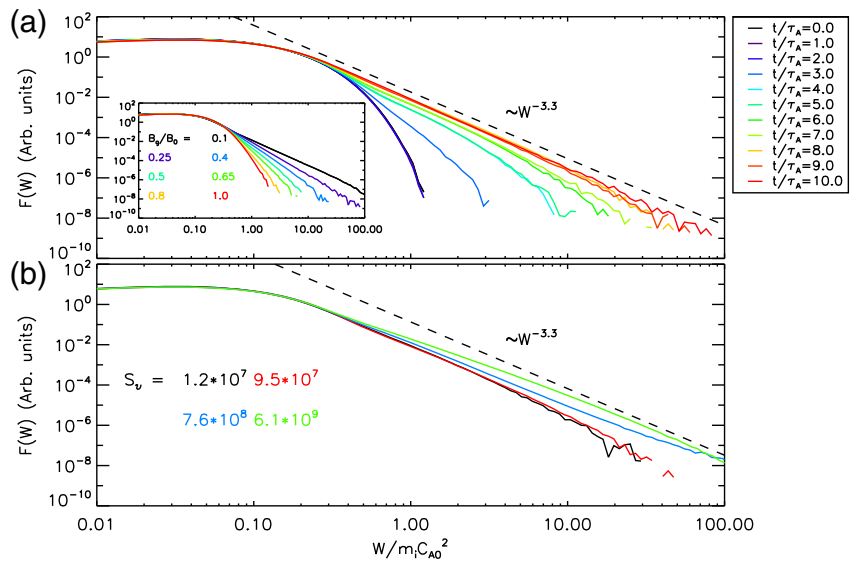

FIG. 2. (a) A log-log plot of $F(W)$ versus energy at multiple times for the $B_{g} / B_{0}=0.25$ simulation. Inset in (a): the late time $F(W)$ for several guide fields. (b) The late time $F(W)$ for $B_{g} / B_{0}=0.25$ with various values of $S_{\nu}$ (effective system size). The dashed line in both (a) and (b) is a power-law with $\delta^{\prime}=3.3$. with reconnected flux so that larger systems produce more extended power laws.

The dependence of $\delta^{\prime}$ on $B_{q}$ is plotted by the black curve in Fig. 3(a). Strong $B_{g}$ produces a soft nonthermal spectrum. The solid red curve is from the theoretical model discussed below. Plotted in Fig. 3(b) is the time dependence of the energy of a typical electron from the power-law tail versus its $x$ position from a simulation with $B_{g} / B_{0}=0.25$. Early in time the electron makes several passes through the system with little change in energy. Once reconnection produces flux ropes, the electron is captured and undergoes Fermi reflection, gaining energy with each bounce, as the flux rope contracts and merges with others. Figure 3(c) is a schematic of the island-merging process that leads to the power-law tail (discussed in the following section).

In exploring the power-law distribution, we averaged over the entire domain to improve the statistics. However, an important question in understanding particle energy gain concerns the relative numbers and energy content of nonthermals (those in the power-law tail) versus those that display a thermal or nearly thermal distribution. The observations suggest that the nonthermals often contain more energy than the thermals in large flares $[4,23,24]$. To explore these questions we analyzed data from more limited spatial domains that include both hot thermal and nonthermal electrons but exclude electrons that have not yet gained energy from reconnection. We focus, therefore, on the interior of magnetic islands where the electron temperature has increased and where there are significant numbers of nonthermal electrons. The goals are to establish the fraction of electrons that can be categorized as thermal versus nonthermal and whether a characteristic effective temperature is associated with the thermal population.

Specifically, we explore the region between the two white ellipses within the middle flux rope in Fig. 1(c). In
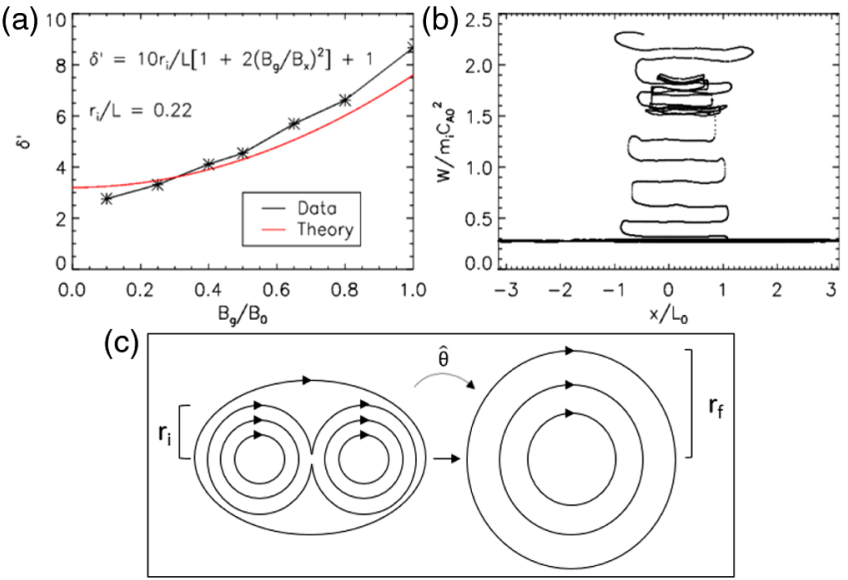

FIG. 3. (a) $\delta^{\prime}$ (black) versus $B_{g}$ and the theoretical fit (red). (b) Energy versus $x$ position of a nonthermal electron. (c) A schematic depicting the flux rope merging mechanism that leads to electron power-law distributions. 

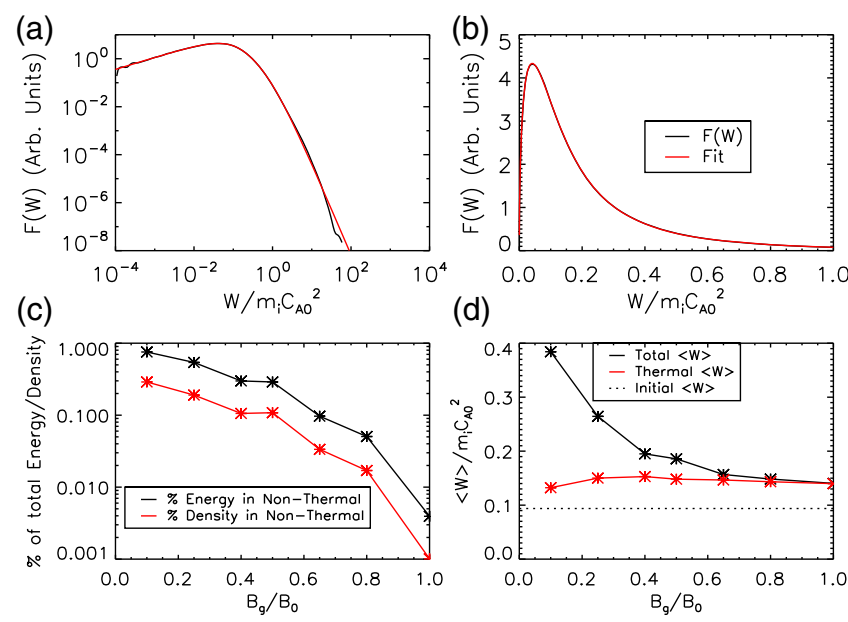

FIG. 4. (a) $F(W)$ (black) along with the fit (red) described in Ref. [25] versus energy on a log-log scale. (b) The same data on a linear-linear scale, zoomed in to low energies to reveal the thermal electrons. (c) The percentage of energy (black) and density (red) of nonthermals versus guide field. (d) the average energy per particle of particle electrons (black) and thermals (red) versus $B_{g}$. The dotted line is the energy from the initial Maxwellian distribution of particle electrons.

Fig. 4(a) we show $F(W)$ (black). The high-energy electrons form a power-law distribution even in this localized region within a single flux rope. In Fig. 4(b) we display the same data, but on a linear-linear scale focused on the lower energies. These two plots reveal that localized regions within magnetic islands contain a mixture of electrons with a range of energies so that characteristics of the thermals and nonthermals can be explored.

To model the distributions in Fig. 4 we use the sum of a Maxwellian and a kappa distribution. The kappa distribution fits the power-law tail of nonthermal electrons and the Maxwellian supplements the Maxwellian component of the kappa distribution at low energies, producing a good fit to the thermal electrons. The fitting procedure is discussed in detail in the Supplemental Material [25]. The outputs of the fit are the spectral index of the nonthermals, and the number density and total energy content of the nonthermals and thermals.

The fitting results for all $B_{g}$ appear in Figs. 4(c) and 4(d). Shown in 4(c) is the percentage of the total density (red) and energy (black) of the nonthermals as defined in Ref. [25] as a function of the guide field. Each distribution came from a region within an island similar to the one shown in Fig. 1(c). For small $B_{g}$ the energy content of the nonthermals is $\sim 80 \%$ of the total particle electron energy and $\sim 20 \%$ of the total electron particle density. As $B_{g}$ increases, the number of nonthermals and their energy content becomes small. In 4(d) is the total energy per particle of the particle electrons (black) and the corresponding energy per particle, or $(3 / 2) T_{t h}$, of the thermals (red), with the initial energy shown as a dotted line. This is further evidence that the nonthermals dominate the total electron energy at low $B_{g}$ where the Fermi drive is strong. On the other hand, the energy of the thermals is relatively insensitive to $B_{g}$ and is likely controlled by slow shocks (see Ref. [25]) rather than by Fermi reflection.

An analytic model for nonthermal electron acceleration.-We present a model for electron acceleration in a current layer with merging magnetic flux ropes that captures the essential results of the simulations, including an expression for the power-law index of the nonthermals and its dependence on the ambient guide field. The model includes the convective loss of electrons injected into large, inactive flux ropes.

The model is based on electron energy gain during the merging of flux ropes. The dominant heating, parallel to the local magnetic field [27,28], results from the shortening of field lines during mergers [12] as shown in Fig. 3(c): merging field lines contract from the figure-eight configuration on the left to the circle on the right. Parallel heating results from the invariance of the parallel action $\oint v_{\|} d l$. Thus, the change in the energy can be calculated by evaluating the geometry of the magnetic field before and after the merger. The calculation presented in the Supplemental Material [25], results in the rate of energy gain

$$
\underline{W}=\frac{d}{d t} W=W \frac{g}{\tau_{r}},
$$

with $\tau_{r} \sim r_{i} / R c_{A x}$ the merger time of a flux rope of initial radius $r_{i}$, where $R \sim 0.1$ is the normalized merger rate, and $c_{A x}$ is the Alfvén speed based on the reconnecting magnetic field $B_{x}$. The factor $g=\left(1+2 B_{g}^{2} / B_{x}^{2}\right)^{-1}$ arises from the dependence of the curvature of the reconnecting field on $B_{g}$ $[11,27,29]$. With the energy gain in Eq. (1), an equation can be derived for the number density $F(x, W, t)$ of electrons per unit energy undergoing reconnection-driven acceleration in a one-dimensional current layer while experiencing convective loss,

$$
\frac{\partial}{\partial t} F+\frac{\partial}{\partial x} v_{x}(x) F+\frac{\partial}{\partial W} W F-D \frac{\partial^{2}}{\partial x^{2}} F=\frac{1}{\tau_{\text {up }}} F_{\text {up }}
$$

where $v_{x}(x)$ describes the convective loss of electrons ejected at the Alfvén speed out of the current layer. We also include a simple constant diffusion within the current layer. The electrons are injected with an initial distribution $F_{\text {up }}$ which is taken as a low-temperature Maxwellian. Although the simulations are periodic and particles are therefore not lost, the large flux ropes that emerge at late time and no longer participate in reconnection act as sinks for energetic electrons $[14,30]$. For a low upstream temperature and strong diffusion $D$, the steady state solution to Eq. (2) is

$$
F_{0} \propto \frac{1}{W} W^{-c_{A x} \tau_{r} / g L} \sim W^{-\left(1+r_{i} / g R L\right)}
$$


The energetic electron spectrum is a power law with a spectral index dependent on the rate of reconnection $R$, the ratio of the characteristic radius $r_{i}$ to the half-width $L$ of the current layer and the strength of $B_{g}$. A strong guide field for which $g \sim\left(2 B_{g}^{2} / B_{x}^{2}\right)^{-1}$ produces a very soft spectrum. This scaling relation is compared with data from our simulations in Fig. 3(a). The best fit to the data has $r_{i} / L=0.22$, consistent with the typical scale of islands in the simulations.

Comparison with observations.-The standard model for a solar flare comes from Ref. [31]. It includes an erupting flux rope that produces a large reconnecting current sheet above a cusp-shaped flare arcade. As reconnection proceeds, more small flux ropes are produced in the current sheet and flow up toward the erupting flux rope, or down toward the arcade. The solar flare of September 10, 2017, was observed by several instruments [32-35]. The gyrosynchrotron spectrum revealed relativistic electrons throughout the reconnecting current sheet, with an increase in intensity where the current sheet met the arcade. The observed power-law indices $\delta^{6}$ for this region fell in the range 3.5-6.5, depending on the position. RHESSI observations for this event revealed both a footpoint and an extended coronal source [32]. The coronal source had a photon spectral index near 4.4, which for thin target emission corresponds to a particle spectral index $\delta^{\prime}=3.9$. Late in the flare the temperature of the hot thermal electrons in the current sheet was analyzed with the EIS Fe XXIV/Fe XXIII ratio [33] and found to have a broad peak near $2.5 \mathrm{keV}$.

Our simulations reveal that the spectral index of nonthermal electrons and the number of the nonthermals depend strongly on the ambient guide field. Comparisons of MHD simulations of the September 10, 2017, flare with gyrosynchrotron emission of energetic electrons revealed that the guide field was $30 \%$ of the reconnecting magnetic field [34]. Based on the data from Fig. 2, our simulations predict a power-law index near 3.5, within the range measured from gyrosynchrotron emission and close to the RHESSI measurement.

We can also compare the temperature jump of the hot thermal electrons in our simulation with the measured $2.5 \mathrm{keV}$ data from EIS. The simulation data in Fig. 4(d) suggest that the hot thermal electrons should have a temperature jump near $0.04 m_{i} C_{A 0}^{2}$. The Alfvén speed was estimated as 6000 to $10000 \mathrm{~km} / \mathrm{s}$ [34], which yields a temperature of $15-42 \mathrm{keV}$, well above the observations. However, such high flow speeds were not measured. At the time of the EIS observations the measured outflow speed was $\sim 800 \mathrm{~km} / \mathrm{s}$ [36], which is likely a lower bound due to projection effects. In situ measurements of reconnecting current sheets at $1 \mathrm{AU}$ indicate that the outflow speed is around $2 / 3$ of the upstream Alfvén speed [37]. Using $1200 \mathrm{~km} / \mathrm{s}$ for $C_{A 0}$, we calculate a hot electron temperature of $0.6 \mathrm{keV}$, which is smaller than the EIS measurement. More precise measurements of the outflow speed are needed.
Observations of large numbers of flares have revealed that the energy in nonthermal electrons exceeds that of the thermal electrons in $\sim 80 \%$ of events, suggesting that solar flares are extremely efficient accelerators [23]. The efficiency of nonthermal electron acceleration was greatest in large flares [24] although recent evidence from NuSTAR suggests that such results might extend to smaller flares [38]. Such results are consistent with Fig. 4(c) if $B_{g} / B_{0}<0.4$. The simulations [Fig. 2(b)] further suggest that even small flares might be efficient sources of nonthermal electrons.

An important question is why conventional PIC modeling has been unable to produce the extended power-law spectra that develop in kglobal. Some groups, e.g., Refs. [39] and [14], have produced modest power laws that extend at most a decade in energy and do not establish the dependence on $B_{g}$. We suggest that these short power laws are due to inadequate separation between kinetic scales and the macrosystem scale. As electrons gain energy from Fermi reflection in the PIC model, their Larmor radii become comparable to the reconnecting flux ropes and they become unmagnetized [14]. This suppresses energy gain associated with Fermi reflection [12]. In PIC simulations the problem is extreme because the macroscale in flares is of the order of $10^{4} \mathrm{~km}$ while the simulation domains are of the order of $10^{2} \mathrm{~m}$. The artificially large electron masses in PIC models further exacerbate the problem. In a real system the Larmor radii of electrons with energies of tens of $\mathrm{MeV}$ remain small so demagnetization in flux ropes is likely unimportant. In kglobal the reconnecting flux ropes are at the macroscale and the electron dynamics is guiding center and therefore fully magnetized.

The collaboration leading to these results was facilitated by the NASA Drive Science Center on Solar Flare Energy Release (SolFER), Grant No. 80NSSC20K0627. We would like to thank Dr. W. Daughton and participants in the NASA Drive Center SolFER for invaluable discussions that contributed to this work. This work has been supported by NSF Grants No. PHY1805829 and No. PHY1500460 and the FIELDS team of the Parker Solar Probe (NASA Contract No. NNN06AA01C) and the FINESST Grant No. 80NSSC19K1435. Fan Guo acknowledges support in part from NASA Grant No. 80NSSC20K1318 and Astrophysics Theory Program, and DOE support through the LDRD program at LANL. Joel Dahlin was supported by an appointment to the NASA Postdoctoral Program at the NASA Goddard Space Flight Center, administered by Universities Space Research Association under contract with NASA. The simulations were carried out at the National Energy Research Scientific Computing Center (NERSC). The data used to perform the analysis and construct the figures for this Letter are preserved at the NERSC High Performance Storage System and are 
available upon request. E. K. acknowledges financial support from the STFC Consolidated Grant No. ST/T000422/1. M. S. acknowledges support in part from NASA Grant No. 80NSSC20K1277.

[1] R. P. Lin and H. S. Hudson, 10-100 keV electron acceleration and emission from solar flares, Sol. Phys. 17, 412 (1971).

[2] A. G. Emslie, H. Kucharek, B. R. Dennis, N. Gopalswamy, G. D. Holman, G. H. Share et al., Energy partition in two solar flare/CME events, J. Geophys. Res. 109, A10104 (2004).

[3] A. G. Emslie, B. R. Dennis, G. D. Holman, and H. S. Hudson, Refinements to flare energy estimates: A followup to "Energy partition in two solar flare/CME events" by A. G. Emslie, B. R. Dennis, G. D. Holman, and H. S. Hudson, J. Geophys. Res. 110, A11103 (2005).

[4] A. G. Emslie, B. R. Dennis, A. Y. Shih, P. C. Chamberline, R. A. Mewaldt, and C. S. Moore, Global energetics of thirtyeight large solar eruptive events, Astrophys. J. 759, 71 (2012).

[5] D. Heristchi and T. Amari, Solar flare high-energy electron spectra, Sol. Phys. 142, 209 (1992).

[6] S. Krucker, H. S. Hudson, L. Glesener, S. M. White, S. Masuda, J.-P. Wuelser, and R. P. Lin, Measurements of the coronal acceleration region of a solar flare, Astrophys. J. 714, 1108 (2010).

[7] M. Oka, S. Ishikawa, P. Saint-Hilaire, S. Krucker, and R. P. Lin, Kappa distribution model for hard $\mathrm{X}$-ray coronal sources of solar flares, Astrophys. J. 764, 6 (2013).

[8] S. Krucker and M. Battaglia, Particle densities within the acceleration region of a solar flare, Astrophys. J. 780, 107 (2014).

[9] E. N. Parker, Sweet's mechanism for merging magnetic fields in conducting fluids, J. Geophys. Res. 62, 509 (1957).

[10] Y. Lin and L. C. Lee, Structure of the dayside reconnection layer in resistive MHD and hybrid models, J. Geophys. Res. Space Phys. 98, A3 (1993).

[11] J. F. Drake, M. Swisdak, H. Che, and M. Shay, Electron acceleration from contracting magnetic islands during reconnection, Nature (London) 443, 553 (2006).

[12] J. F. Drake, M. Swisdak, and R. Fermo, The power-law spectra of energetic particles during multi-island magnetic reconnection, Astrophys. J. Lett. 763, L5 (2013).

[13] F. Guo, H. Li, W. Daughton, and Y. Liu, Formation of Hard Power-Laws in the Energetic Particle Spectra Resulting from Relativistic Magnetic Reconnection, Phys. Rev. Lett. 113, 155005 (2014).

[14] X. Li, F. Guo, H. Li, A. Stanier, and P. Kilian, Formation of power-law electron energy spectra in three-dimensional low- $\beta$ magnetic reconnection, Astrophys. J. 884, 118 (2019).

[15] R. L. Fermo, J. F. Drake, and M. Swisdak, A statistical model of magnetic islands in a current layer, Phys. Plasmas 17, 010702 (2010).

[16] J. F. Drake, H. Arnold, M. Swisdak, and J. T. Dahlin, A computational model for exploring particle acceleration during reconnection in macroscale systems, Phys. Plasmas 26, 012901 (2019).
[17] H. Arnold, J. Drake, M. Swisdak, and J. Dahlin, Large-scale parallel electric fields and return currents in a global simulation model, Phys. Plasmas 26, 102903 (2019).

[18] J. Egedal, W. Daughton, and A. Le, Large-scale electron acceleration by parallel electric fields during magnetic reconnection, Nat. Phys. 8, 321 (2012).

[19] J. Egedal, W. Daughton, A. Le, and A. L. Borg, Double layer electric fields aiding the production of energetic flat-top distributions and superthermal electrons within magnetic reconnection exhausts, Phys. Plasmas 22, 101208 (2015).

[20] C. C. Haggerty, M. A. Shay, J. F. Drake, T. D. Phan, and C. T. McHugh, The competition of electron and ion heating during magnetic reconnection, Geophys. Res. Lett. 42, 9657 (2015).

[21] R. P. Lin, S. Krucker, G. J. Hurford, D. M. Smith, H. S. Hudson, G. D. Holman, R. A. Schwartz, B. R. Dennis, G. H. Share, R. J. Murphy, A. G. Emslie, C. Johns-Krull, and N. Vilmer, RHESSI observations of particle acceleration and energy release in an intense solar gamma-ray line flare, Astrophys. J. 595, L9 (2003).

[22] P. K. Kaw, E. J. Valeo, and P. H. Rutherford, Tearing Modes in a Plasma with Magnetic Braiding, Phys. Rev. Lett. 43, 1398 (1979).

[23] M. Aschwanden, G. Holman, A. O’Flannagain, A. Caspi, J. McTiernan, and E. Kontar, Global energetics of solar flares. III. Nonthermal energies, Astrophys. J. 832, 27 (2016).

[24] A. Warmuth and G. Mann, Constraints on energy release in solar flares from RHESSI and GOES x-ray observations, Astron. Astrophys. 588, A115 (2016).

[25] See Supplemental Material at http://link.aps.org/ supplemental/10.1103/PhysRevLett.126.135101 for a discussion on electron heating, a description of how we fit the electron distribution function, and a description of our analytic model for calculating the power law index of the electrons, which includes Ref. [26].

[26] Q. Zhang, J. F. Drake, and M. Swisdak, Instabilities and turbulence in low- $\beta$ guide field reconnection exhausts with kinetic Riemann simulations, Phys. Plasmas 26, 102115 (2019).

[27] J. T. Dahlin, J. F. Drake, and M. Swisdak, The role of threedimensional transport in driving enhanced electron acceleration during magnetic reconnection, Phys. Plasmas 24, 092110 (2017).

[28] J. T. Dahlin, J. F. Drake, and M. Swisdak, The mechanisms of electron heating and acceleration during magnetic reconnection, Phys. Plasmas 21, 092304 (2014).

[29] J. T. Dahlin, J. F. Drake, and M. Swisdak, Parallel electric fields are inefficient drivers of energetic electrons in magnetic reconnection, Phys. Plasmas 23, 120704 (2016).

[30] J. T. Dahlin, J. F. Drake, and M. Swisdak, Electron acceleration in three-dimensional magnetic reconnection with a guide field, Phys. Plasmas 22, 100704 (2015).

[31] J. Lin and T. G. Forbes, Effects of reconnection on the coronal mass ejection process, J. Geophys. Res. 105, 2375 (2000).

[32] D. E. Gary, B. Chen, B. R. Dennis, G. D. Fleishman, G. J. Hurford, S. Krucker, J. M. McTiernan, G. N. Nita, A. Y. Shih, and S.M. White, Microwave and hard X-ray 
observations of the 2017 september 10 solar limb flare, Astrophys. J. 863, 83 (2018).

[33] H. P. Warren, D. H. Brooks, I. Ugarte-Urra, J. W. Reep, N. A. Crump, and G. A. Doschek, Spectroscopic observations of current sheet formation and evolution, Astrophys. J. 854, 122 (2018).

[34] B. Chen, C. Shen, D. Gary, K. Reeves, G. Fleishman, S. Yu, F. Guo, S. Krucker, J. Lin, G. Nita, and X. Kong, Measurement of magnetic field and relativistic electrons along a solar flare current sheet, Nat. Astron. Lett. 4, 1140 (2020).

[35] G. D. Fleishman, D. E. Gary, B. Chen, N. Kuroda, S. Yu, and G. M. Nita, Decay of the coronal magnetic field can release sufficient energy to power a solar flare, Science $\mathbf{3 6 7}$, 278 (2020).
[36] X. Cheng, Y. Li, L.F. Wan, M. D. Ding, P. F. Chen, J. Zhang, and J. J. Liu, Observations of Turbulent magnetic reconnection within a solar current sheet, Astrophys. J. 866, 64 (2018).

[37] C. Haggerty, M. Shay, A. Chasapis, T. Phan, J. F. Drake, K. Malakit, P. Cassak, and R. Kieokaew, The reduction of magnetic reconnection outflow jets to sub-Alfvénic speeds, Phys. Plasmas 25, 102120 (2018).

[38] L. Glesener, S. Krucker, J. Duncan, I. Hannah, B. Grefenstette, B. Chen, D. Smith, S. White, and Hudson, Accelerated electrons observed down to $<7 \mathrm{keV}$ in a NuSTAR solar microflare, Astrophys. J. Lett. 891, L34 (2020).

[39] G. Baumann, T. Haugbølle, and $\AA$ Nordlun, Kinetic modeling of particle acceleration in a solar null-point reconnection region, Astrophys. J. 771, 93 (2013). 